\title{
VIESES NA SATISFAÇÃO: O EFEITO MODERADOR DA DIFICULDADE E DA OBRIGAÇÃO SOBRE RECORDAÇÕES POSITIVAS E NEGATIVAS
}

\author{
Juan José Camou Viacava \\ juanjcviacava@gmail.com \\ Danielle Mantovani \\ dm_lucena@yahoo.com.br \\ Jose Carlos Korelo \\ korelo@yahoo.com
}
Paulo Henrique Muller Prado
pprado@ufpr.br

Universidade Federal do Paraná - Curitiba, PR

http://dx.doi.org/10.1590/1413-2311.0682014.54828

Recebido em 01/12/2014

Aprovado em 17/07/2015

Disponibilizado em 01/08/2015

Avaliado pelo sistema "double blind review"

Revista Eletrônica de Administração

Editor: Luís Felipe Nascimento

ISSN 1413-2311 (versão "on line")

Editada pela Escola de Administração da Universidade Federal do Rio Grande do Sul.

Periodicidade: Quadrimestral

Sistema requerido: Adobe Acrobat Reader.

\section{RESUMO}

Baseado na premissa teórica de que os indivíduos podem distorcer informações e opiniões no processo de recuperação de suas memórias, este artigo propõe que as avaliações de satisfação são influenciadas por tais distorções. Em dois experimentos foi requisitado que indivíduos recordassem e relatassem fatos sobre empresas provedoras de serviços, demonstrando que é possível influenciar positivamente (vs. negativamente) avaliações de satisfação solicitando apenas fatos positivos (vs. negativos). Entretanto, a influência da valência positiva ou negativa não age de modo isolado, mas depende de dois outros fatores em conjunto, a dificuldade e a obrigatoriedade percebida. Em atos volitivos (com intenção, sem obrigação), indivíduos que tiveram que recordar eventos positivos (vs. negativos) de seus relacionamentos comerciais avaliaram-se como mais (vs. menos) satisfeitos, mas apenas quando a dificuldade percebida foi maior, indicando maior deliberação sobre os eventos. Contudo, quando obrigados a recordar e difundir informações esta influência de diferentes valências ocorreu apenas quando a tarefa foi percebida como mais fácil. Desta forma, este estudo contribui para teorias de marketing de relacionamento ao tratar de influencias sobre um importante fator, a satisfação e, para teorias de processamento de informações e decisão ao demonstrar que o 
VIESES NA SATISFAÇÃO: O EFEITO MODERADOR DA DIFICULDADE E DA OBRIGAÇÃO SOBRE RECORDAÇÕES POSITIVAS E NEGATIVAS

enviesamento de avaliações não depende apenas da dificuldade percebida, mas sim atua de forma conjunta com a obrigatoriedade percebida na tarefa.

Palavras-Chave: Satisfação; Vieses; Memória; Julgamentos; Dificuldade; Volição.

\title{
BIASES IN SATISFACTION: THE MODERATOR EFFECT OF DIFFICULTY AND OBLIGATION ON POSITIVE AND NEGATIVE MEMORIES
}

\begin{abstract}
Based on the theoretical assumption that individuals can distort information and opinions in their memories recovery process, this article proposes that satisfaction evaluations are influenced by such distortions. In two experiments individuals were requested to remind and to report facts about service providers companies, demonstrating that it is possible to positively influence (vs. negative) satisfaction evaluations by asking only positive facts (vs. negative). However, the influence of positive or negative valence does not act isolated, but depends on two other factors together, the difficulty and the obligation perceived. In volitional acts (with intention, not forced) individuals who had to recall positive events (vs. negative) of their business relationships evaluated themselves as more (vs. less) satisfied, but only when the perceived difficulty was higher, indicating more deliberation on the events. However, when forced to remember and disseminate information, the different valences influence only occurred when the task was perceived as easier. Therefore, this study contributes to relationship marketing theories by investigating the influence on an important factor, satisfaction evaluation and, contributes to information processing and decision theories by demonstrating that bias of evaluations depends not only of the perceived difficulty, but also of the perceived obligation on the task.
\end{abstract}

Keywords: Satisfaction; Biases; Memory; Judgments; Difficulty; Volition.

\section{SESGOS DE SATISFACCIÓN: EL EFECTO MODERADOR DE DIFICULTAD Y OBLIGACIÓN DE RECUERDOS POSITIVOS Y NEGATIVOS}

\section{RESUMEN}

Partiendo del supuesto teórico de que los individuos pueden destorcer la información y opiniones en el proceso de recuperación de sus recuerdos, este artículo propone que las evaluaciones de satisfacción son influenciadas por estas distorsiones. En dos experimentos fue pedido que individuos recordasen y reportasen datos sobre empresas de servicios, demostrando que es posible influir positivamente (vs. negativamente) la satisfacción pidiendo solamente memorias positivas (vs. negativas). Sin embargo, la influencia de la valencia positiva o negativa no actúa de manera aislada, sino que depende de otros dos factores, la dificultad y la obligación percibida. En los actos volitivos (de voluntad), las personas que tuvieron que recordar acontecimientos positivos (vs. negativos) de sus relaciones comerciales se sintieron más (vs. menos) satisfechos, pero sólo cuando la dificultad percibida fue mayor. Eso indica que tuvieron que pensar más sobre los acontecimientos, procesando más la 
información. Sin embargo, cuando se vieron obligados a recordar y difundir la información, esta influencia de las diferentes valencias se produjo sólo cuando la tarea fue percibida como más fácil. De esta manera, este estudio contribuye a las teorías de marketing de relacionamiento cuando se trata de la influencia de un factor importante, la satisfacción y, para las teorías de procesamiento de información y de decisiones al demostrar que las distorsiones en las evaluaciones dependen no sólo de la dificultad percibida, mas conjuntamente con la obligación percibida en la tarea.

Palabras Clave: Satisfacción; Sesgos; Memoria; Juicios; Dificultad; Volición.

\section{INTRODUÇÃO}

"Satisfação é a resposta ao consumidor de algo realizado. É um julgamento que um produto ou uma característica do serviço, ou do produto ou serviço em si, forneceu (ou está oferecendo)...” (OLIVER, 1997, p.13).

A satisfação é apontada como processo crítico a ser compreendido impactando no comprometimento, confiança e lealdade (FORNELL, JOHNSON E ANDERSON, 1994). A satisfação é relacionada positivamente ao bom desempenho comercial em quase todos os segmentos (OLIVER, 2010; SIDDIGI, 2011). Por isso modelos como o cognitivo (OLIVER, 1980) e o cognitivo-afetivo (OLIVER, 1993) de satisfação são amplamente utilizados.

Em ambos os modelos existe uma formação global de avaliações e julgamentos entre informações positivas e negativas resgatadas na memória, ou ainda externas como informações de boca-a-boca. Entretanto, recordações e julgamentos são usualmente distorcidas (KAHNEMAN, 2011). Estes processos são influenciados por fatores situacionais como: tempo; dificuldade; esforços/energia necessários; o modo que informações são descritas (BETTMAN, LUCE E PAYNE, 1998); expectativas de qualidade e de desempenho, necessidade de impor responsabilidades e justificação (OLIVER, 2010), além de distorções ou vieses em codificar, armazenar e recuperar informações (KAHNEMAN, 2011). A importância do assunto é destacada na literatura de marcas, em que a codificação e recuperação de informações é uma das bases para a lembrança, reconhecimento e assim reduzir riscos nas decisões de compra (KELLER, 2006; AAKER, 2007). Para Kahneman (2011), quanto mais fácil o resgate de informações, mais fáceis serão os julgamentos para o indivíduo. Como resultado indireto, o indivíduo percebe tais informações como "mais corretas" e têm maior 
VIESES NA SATISFAÇÃO: O EFEITO MODERADOR DA DIFICULDADE E DA OBRIGAÇÃO SOBRE RECORDAÇÕES POSITIVAS E NEGATIVAS

confiança sobre elas, mesmo que a informação tenha sido codificada ou recuperada de forma incorreta.

Este artigo propõe demonstrar em 2 experimentos as relações entre a memória e as avaliações de satisfação, além de formas de alterar estas avaliações. Como outros autores, demonstramos que as avaliações podem ser distorcidas ao focar na recordação de valências específicas (AARTS E DIJKETRHUIS, 1999; WINKIELMAN E SCHWARZ, 2001; HADDOCK, 2002 SCHARZ, 2004). Demonstramos também o papel de dois moderadores no processo, a dificuldade percebida na tarefa de recordar e fazer o boca-à-boca demonstrada por outros autores (WINKIELMAN E SCHWARZ, 2001; KAHNEMAN, 2011) e, propomos que a obrigatoridade da tarefa (PRITCHARD e outros 1999; BAGOZZI e LEE, 1999; KORIAT e outros, 2008) também atua na moderação.

Estudos utilizando a dificuldade percebida têm demonstrado que a percepção de origem da dificuldade modifica a explicação pessoal-nativa afetando os resultados (SCHWARZ e outros, 1991; WINKIELMAN E SCHWARZ, 2001; SCHWARZ, 2004). Quando o indivíduo percebe ter dificuldades em recordar algo (ex: características de um antigo amigo de infância), ele pensa que seu pensamento está equivocado e afeta seus

julgamentos. Por outro lado, se ele percebe que a tarefa é difícil independente de sua memória (ex: percebe que foi à muito tempo, e a tarefa em si é difícil ou quase impossível) esta influência deixa ser significativa.

Entretanto, apresentamos evidências teóricas e empíricas que o mecanismo de volição (versus obrigatoriedade) também tem um efeito moderador (PRITCHARD e outros 1999; BAGOZZI e LEE, 1999; KORIAT e outros, 2008). Quando indivíduos recordam de fatos sem serem obrigados a isto, o processo de deliberação ligado à maior dificuldade na tarefa leva o indivíduo a dar maior peso no que foi recordado. Entretanto, quando ele é obrigado a recordar algo, a facilidade percebida afeta seus julgamentos de acordo com o que foi recordado.

\section{A FORMAÇÃO DA SATISFAÇÃO, MEMÓRIA E SEUS EFEITOS}

Os modelos cognitivo (OLIVER, 1980) e o cognitivo-afetivo (OLIVER, 1993) propõem que a satisfação é resultado da avaliação subjetiva das expectativas em relação ao desempenho e, fatores de afeto positivo e negativo. Quando o desempenho excede as expectativas resulta em desconfirmação positiva, gerando satisfação. Quando fica aquém resulta em desconfirmação

REAd | Porto Alegre - Edição 81 - № 2 - maio/agosto 2015 - p. 437-461 
Juan José Camou Viacava \& Danielle Mantovani

negativa gerando insatisfação. Quando as expectativas se igualam ao desempenho, existe confirmação e o indivíduo fica saciado. Desta forma o consumidor experencia uma satisfação baseada em comparações e análise de informações. Quando passa a incorporar afeto e sentimentos das experiências às suas expectativas e avaliações de desempenho, resulta em avaliações cognitivo-afetiva de satisfação. Consequentemente, as avaliações de satisfação direcionam decisões de recompra e boca-a-boca (HART E JOHNSON, 1999; KEININGHAM e outros, 2007), e com o tempo se transformam em comprometimento, confiança e leadade (OLIVER, 2010).

Quando informações, ou mesmo “pistas" de eventos são ativadas, as demais informações como causas, resultados e modos de ação vão sendo recordadas, correta ou incorretamente (HOMMEL e outros, 2001; GOMES E OUTROS, 2013; SZPUNAR, ADDIS E SCHACTER 2012). Como resultado, ao escutar o nome de seu provedor de serviços o consumidor primeiro recordará os eventos que ocorreram com ele (KELLER, 2006) e mesmo com outros para formar uma avaliação global de satisfação (OLIVER, 2010).

Entretanto, este processo de recordação geralmente é falho (KAHNEMAN, 2011). Por exemplo, Oliver (1999), Zwick, Baumgartner e Pieters (1995) indicam que os indivíduos podem distorcer lembranças recriando expectativas iniciais para adaptá-las aos resultados das avaliações de satisfação presentes.

Além de tentar "alterar" memórias, o indivíduo pode também alterar suas avaliações presentes nesse processo de recordação e processamento de informações. Para Kahneman (2011), o indivíduo ao se deparar com situações difíceis usualmente emprega uma ou mais estratégias simplificadoras, denominadas heurísticas, para facilitar o processo e diminuir a tensão situacional. Entretanto, ao fazer isto ele enviesa suas decisões e julgamentos, pois altera os pesos dos atributos ou, foca em aspectos mais salientes no momento. Entretanto, a forma como isto ocorre dependerá da explicação "vulgar", pessoal ou compartilhada por um grupo e pela percepção de onde vem a dificuldade (SCHARZ, 2004).

Scharz (2004) aponta que, se o indivíduo perceber que ele tem dificuldade numa tarefa de recordar, ele ficaria com dúvidas e diminuiria sua confiança no assunto. Por outro lado, se perceber que a tarefa em sí é difícil e independe de sua memória ou experiência, ele passaria a processar mais as informações do que recordou dando maior peso nelas.

REAd | Porto Alegre - Edição 81 - N 2 - maio/agosto 2015 - p. 437-461 


\section{VIESES NA SATISFAÇÃO: O EFEITO MODERADOR DA DIFICULDADE E DA} OBRIGAÇÃO SOBRE RECORDAÇÕES POSITIVAS E NEGATIVAS

Em um exemplo de reconstrução do passado afetando avaliações presentes e controlando a percepção de dificuldade (recordar em si era difícil), Winkielman e Schwarz (2001) requisitaram que indivíduos recordassem diferentes quantidades de memórias (12 tarefa difícil vs 4 - tarefa fácil) sobre a infância, e também de diferentes valências (positivas $v s$ negativas), resultando num design $2 \times 2$. Antecipando que recordações negativas são mais fáceis de recordar e manipulando que a dificuldade era parte da tarefa em si (e não de dificuldade de recordar) aplicaram um procedimento de controle de dificuldade entre as valências. Os autores informaram os participantes que recordações da infância vão desaparecendo e são difícieis de recordar. Disseram também que eventos negativos seriam difíceis de ser recordados pois indivíduos procuram esquecê-los, enquanto eventos positivos são esquecidos pois não pensamos muito sobre eles.

Dentro dos grupos de maior número de memórias solicitadas verificaram que indivíduos que recordaram apenas memórias positivas avaliaram suas infâncias como mais felizes do que aqueles que recordaram apenas memórias negativas. Esta diferença não foi significativa dentro do grupo de menor número de memórias solicitadas, em que memórias positivas não tiveram diferenças significativas de memórias negativas, indicando o efeito de interação da dificuldade percebida da tarefa em si e da valência de memórias resgatadas. Resultados semelhantes foram encontrados por Stepper e Strack (1993) e Sanna, Schwarz e Small (2002).

Estes resultados condizem com o efeito de memórias codificadas sobre experiências passadas. Para Kahneman (2011), quanto mais rápido, fácil ou automático uma avaliação é formada, menor é o esforço e menor é a deliberação de informações. Desta forma, indivíduos com maior facilidade percebida estariam apenas resgatando opiniões e avaliações passadas, sem terem que reavaliá-las. Como resultado secundário, também apresentariam maior confiança em suas opiniões.

Assim, quando os indivíduos tinham que recordar poucos pontos, pouco deliberavam sobre eles não indicando diferenças significativas entre as valências requisitadas (WINKIELMAN E SCHWARZ, 2001). Entretanto, quando a dificuldade era maior, mais os indivíduos utilizavam e deliberavam sobre as informações (KAHNEMAN, 2011), procurando por explicações (SCHWARZ, 2001) mudando suas opiniões. Nordgren e Dijksterhuis (2009) também apontam para este efeito em seus experimentos. Ao manipular a dificuldade de 
Juan José Camou Viacava \& Danielle Mantovani

avaliação pela quantidade de quesitos a serem avaliados, causaram maiores variações entre avaliações passadas e presentes. Desta forma propomos a seguinte hipótese:

\section{HIPÓTESE 1: as avaliações de satisfação serão influenciadas pela valência das memórias requisitadas, mas apenas quando a tarefa em si for percebida como difícil.}

Como destacado, a dificuldade da tarefa também pode afetar julgamentos de outras formas se o indivíduo não perceber naturalmente a tarefa em si difícil, mas sim um problema de acessibilidade de memória. Schwarz e outros (1991), Aarts e Dijkterhuis (1999) e Haddock (2002) verificaram que quando indivíduos percebiam a dificuldade como um problema de acessibilidade ou frequência de fatos numa memória, o efeito moderador da dificuldade continuava, mas o padrão dos resultados não. Enquanto Winkielman e Schwarz (2001) verificou que ao deliberar mais sobre fatores positivos ( $v s$ negativas) tornava avaliações mais positivas, estes outros verificaram que, quanto maior número de fatos requisitados, menor era a confiança sobre os fatos recordados e, traziam uma percepção inversa nas avaliações. Por não conseguir apontar/recordar mais fatos negativos (usualmente 12), indivíduos faziam avaliações mais positivas do que indivíduos que tinham que recordar menos fatos negativos (6 fatos). O mesmo padrão acontecia ao requisitar a recordação de fatos positivos. Quanto mais fatos positivos (12 vs 6) menos favoráveis eram as avaliações. Assim, quanto mais fatos assertivos eram requisitados, menos assertivas eram as avaliações (SCHWARZ e outros, 1991). Quanto mais eventos para uso de biciletas fossem necessários, menor era sua percepção de uso (AARTS e DIJKETRHUIS, 1999). Quanto mais pensamentos favoráveis sobre o então $1^{\circ}$ ministro Tony Blair eram necessários, menos favoráveis eram as avaliações dos estudantes britânicos sobre ele (HADDOCK, 2002).

Estas evidências indicam que a percepção de origem da dificuldade modera os efeitos, mas acreditamos também que a percepção de obrigação na atividade tenha efeito semelhante. Para Koriat e outros (2008) quando a recordação de fatos ou motivos para suportar uma opinião são mais espontâneas, a confiança seria maior. Entretanto, quando não é espontânea, gera desconfiança nas próprias recordações e o sentimento que, se "eu não pude completar a tarefa, eu devo estar errado". Desta forma a falta de confiança em encontrar tantos pontos positivos ( $v s$ negativos) também levaria à desconfiança que as avaliações deveriam ser positivas (negativas) (AARTS e DIJKETRHUIS, 1999, HADDOCK, 2002).

REAd | Porto Alegre - Edição 81 - № 2 - maio/agosto 2015 - p. 437-461 
VIESES NA SATISFAÇÃO: O EFEITO MODERADOR DA DIFICULDADE E DA OBRIGAÇÃO SOBRE RECORDAÇÕES POSITIVAS E NEGATIVAS

Apesar da possível influência da confiança ao ter que obrigatoriamente completar uma tarefa, a própria percepção de imposição pode afetá-la diretamente. Num ato de volição, em que o indivíduo faz ou continua um ato enquanto deseje, ele percebe suas ações como mais adequadas em função do objetivo e aumenta seu comprometimento com que faz (PRITCHARD e outros 1999; FISCHBACK e FERGUSON, 2007) intensificando o valor do que faz (HIGGINS e SCHOLER, 2009) ou mesmo sobre a empresa que tem relacionamento escolhido, aumentando seu boca-a-boca (BLOEMER e outros, 2007). Para Bagozzi (1993) a escolha própria estimula outros processos que consistem em planos e esforços voltados para a iniciação, acompanhamento e orientação de busca dos objetivos traçados, legitimando-os. Entretanto, ao ser obrigado a empreender num ato ou continuar nele contra a vontade, pode reduzir seu comprometimento e perceber suas ações como menos adequadas. Outros efeitos neste sentido podem ser vistos como, maior aprendizado sobre um assunto (HAUNSCHILD e RHEE, 2004) e maior aceitação de inovações, ambos quanto maior a percepção de volição (BAGOZZI e LEE, 1999).

Desta forma, propomos que as avaliações de satisfação, focadas em eventos do histórico de relacionamento (OLIVER, 2010), dependem da dificuldade de recordação. Esta dificuldade quando presente num ato volitivo (sem obrigatoriedade) aumenta o comprometimento no que é proposto (PRITCHARD e outros 1999), aumentando também a ruminação e foco no que foi recordado (KAHNEMAN, 2011). Quando não existir espontaneidade (KORIAT e outros, 2008), mas existir obrigatoriedade, consumidores forçados a recordar maior número de recordações positivas (negativas) teriam avaliações menos positivas (negativas) que aqueles forçados a recordar menor número de recordações. Isto poderia ocorrer pela falta de confiança (HADDOCK, 2002, e AARTS e DIJTERHUIS, 1999) e pela falta de comprometimento, já que está contra a sua vontade (BAGOZZI, 1993; FISCHBACK e FERGUSON, 2007; HIGGINS e SCHOLER, 2009). Desta forma, a obrigatoriedade estaria moderando a influencia da dificuldade da tarefa em si (moderando os efeitos na Hipótese 1). Assim propomos:

HIPÓTESE 2: a percepção de obrigatoriedade na tarefa (vs sem obrigação - volitiva) modera o efeito da dificuldade e valências recordadas sobre as avaliações de satisfação. 


\section{MÉTODO}

O método de pesquisa adotado é o de abordagem quantitativa, de classificação causal e de natureza experimental (KEPPEL E WICKIN, 2004; GOODWIN, 2008). Desta forma, procura a partir da manipulação de variáveis independentes (VI: dificuldade e; obrigatoriedade da tarefa) verificar os seus efeitos sobre a variável dependente (VD: avaliação de satisfação).

Para alcançar o objetivo do estudo foi então construído um modelo do fenômeno reduzindo-o num conjunto parcimonioso de variáveis que pudessem ser medidas e observadas, e devidamente controladas através de projeto e análise estatística (GOODWIN, 2008).

A coleta de dados foi do tipo não-probabilística por conveniência, composta por estudantes de graduação. Os objetivos, descrição e resultados de cada estudo são descritos individualmente a seguir.

\subsection{Estudo 1}

\subsubsection{Amostra e procedimentos}

Para verificar a hipótese 1, um experimento foi realizado com 159 alunos de graduação pela internet (50,9\% homens, idade média 29 anos). O experimento consistiu numa adaptação da manipulação de Winkielman e Schwarz (2001) de resgate, e reporte, de fatos e/ou memórias positivas e negativas. Ao invés de ser utilizadas fatos e/ou memórias sobre a infância, foram solicitados fatos sobre a operadora de telefonia celular do respondente, que consideramos análoga ao comportamento de boca-a-boca. Também foi modificada a manipulação de controle de dificuldade, por uma proposta mais prática de limitação de tempo (BETTMAN e outros, 1998).

O design do experimento resultou num factorial design com duas variáveis independentes manipuladas, gerando um design 2 (VI - fatos: 'positivos' vs 'negativos') x 2 (VI - dificuldade pela quantidade de fatos/pontos:12 - difícil vs 6 - fácil). Os respondentes foram convidados por e-mail a participar de uma pesquisa sobre operadoras de celular. $\mathrm{Na}$ primeira página da pesquisa preenchiam dados pessoais e sócio-demográficos. Para controlar a dificuldade da tarefa, eram informados que teriam um limite de 3 minutos para completar a tarefa seguinte. Ao limitar o tempo, a tarefa em si seria vista como difícil (BETTMAN e outros, 1998), minimizando o efeito de acessibilidade de memória (SCHWARZ, 2001).

REAd | Porto Alegre - Edição 81 - Nº 2 - maio/agosto 2015 - p. 437-461 


\section{VIESES NA SATISFAÇÃO: O EFEITO MODERADOR DA DIFICULDADE E DA} OBRIGAÇÃO SOBRE RECORDAÇÕES POSITIVAS E NEGATIVAS

Em seguinda os respondentes eram direcionados aleatoriamente a uma das 04 (quatro) condições (12 pontos/fatos positivos, 12 pontos/fatos negativos, 6 pontos/fatos positivos ou 6 pontos/fatos negativos). Mesmo com a requisição de um número pré-determinado de pontos/fatos (exemplo: enunciado de 12 positivos: "Por favor, aponte 12 pontos/fatos/ocorrências POSITIVOS(as) sobre sua operadora de telefonia celular.") os respondentes não eram obrigados completá-los para continuar a pesquisa, e tampouco esperar os 3 minutos. Apenas 3 respondentes não apresentaram qualquer fato/ponto sobre sua operadora celular e foram retirados da amostra.

Depois de apontar os fatos sobre a operadora respondiam 04 quesões sobre satisfação com sua operadora de celular. Três foram adaptadas de Fornell e outros (1994) e Bettencourt, 1997 (satisfação geral - "Como você avaliaria sua satisfação no relacionamento com a sua empresa de telefonia celular?", ancorada em "1-muito insatisfeito" e "10-muito satisfeito"; distância das expectativas - "Como você avaliaria o relacionamento com a sua empresa de telefonia celular conforme suas expectativas?" ancorada em "1-muito abaixo das expectativas" e "10-muito acima das expectativas" e; desconformidade global - "Como você avaliaria o seu relacionamento com a empresa de telefonia celular?" ancoradas em "1-muito longe do ideal" e “10-muito próximo do ideal”) e uma questão de avaliação afetiva de Garbarino e Jonhson (1999) (“Avalie se você concorda com a afirmação: "Eu gostaria muito de continuar comprando e me relacionando com minha empresa de telefonia celular."” ancorada em "1-discordo totalmente" e "10-concordo totalmente") - todas traduzidas e utilizadas por Prado e Santos (2004).

Em seguida, respondiam sobre a dificuldade percebida em apontar os pontos/fatos (ancorada em "1 - muito fácil" e "10 - muito difícil") e a confiança sobre o relato dos pontos/fatos que aconteceram ("Indique a porcentagem de confiança geral de que você relatou os fatos exatamente como acontece/ocorreram com você”).

\subsubsection{Checagem das manipulações}

Os resultados das checagens foram considerados satisfatórios. Testes $t$ indicaram maior número de fatos/pontos apontados para o grupo com 12 pontos $(\mathrm{n}=79)$ do que para o grupo de 6 pontos $(\mathrm{n}=80)\left(t=2,002 p=0,047 ; \mathrm{M}_{12 \text { pontos }}=5,31 \mathrm{DP}=3,10 ; \mathrm{M}_{6 \mathrm{pontos}}=4,52 \mathrm{DP}=1,68\right)$ e maior dificuldade em apontar fatos $\left(t=3,501 p<0,001 ; \mathrm{M}_{12 \text { pontos }}=6,53 \mathrm{DP}=2,57 ; \mathrm{M}_{6 \mathrm{pontos}}=5,05\right.$ $\mathrm{DP}=2,76)$, mas sem diferenças para a confiança dos fatos relatados $(t=0,116 p=0,908$; 
$\left.\mathrm{M}_{12 \text { pontos }}=76,79 \mathrm{DP}=21,58 ; \quad \mathrm{M}_{6 \mathrm{pontos}}=76,39 \mathrm{DP}=22,27\right)$ indicando que ao manipular a quantidade de pontos a ser relatados, não foi alterada a confiança como nos experimentos de Haddoc (2002) e Aarts e Dijkterhuis (1999) e que naqueles responderia como explicação.

Testes $t$ indicaram que respondentes que tiveram que apontar apenas pontos positivos $(\mathrm{n}=80) \quad$ indicaram menos pontos/fatos $\left(t=2,799 \quad p=0,006 ; \quad \mathrm{M}_{\text {pontospositivos }}=4,37 \quad \mathrm{DP}=2,45\right.$; $\left.\mathrm{M}_{\text {pontosnegativos }}=5,46 \mathrm{DP}=2,47\right)$, perceberam maior dificuldade em relatar fatos/pontos do que aqueles que apontaram apenas pontos negativos $(\mathrm{n}=79)\left(t=5,814 p<0,001 ; \mathrm{M}_{\text {pontospositivos }}=6,94\right.$ $\left.\mathrm{DP}=2,45 ; \mathrm{M}_{\text {pontosnegativos }}=4,62 \mathrm{DP}=2,56\right)$ e também menor confiança sobre o que relataram $\left(t=2,314 p=0,022 ; \mathrm{M}_{\text {pontospositivos }}=72,67 \mathrm{DP}=23,08 ; \mathrm{M}_{\text {pontosnegativos }}=80,61 \mathrm{DP}=19,87\right)$. Estes resultados corrobram com outros autores que, informações de valência negativa sejam mais recordáveis, mais fáceis de apontar e tenham assim maior confiança atrelada (KANOUSE e HANSON, 1972; KANOUSE, 1984; WINKIELMAN e SCHWARZ, 2001; e BRAINERD e outros, 2010; KAHNEMAN, 2011).

Como checagem indireta, uma análise do tempo utilizado para preencher os pontos/fatos requisitados demonstrou um efeito da valência $\left(F(1,157)=5,416, \eta_{p}{ }^{2}=0,029\right.$ $\mathrm{p}=0,021 ; \mathrm{M}_{\text {positivos }}=83,82$ segundos $\mathrm{DP}=57,71 / \mathrm{M}_{\text {negativos }}=65,17$ segundos $\left.\mathrm{DP}=51,96\right)$ e da manipulação de dificuldade $\left(F(1,157)=6,814, \quad \eta_{\mathrm{p}}{ }^{2}=0,036 \quad p=0,010 ; \quad \mathrm{M}_{12 \text { pontos/fatos }}=83,99\right.$ segundos $\mathrm{DP}=60,42$ / $\mathrm{M}_{6 \mathrm{fatos} / \text { pontos }}=63,82$ segundos $\left.\mathrm{DP}=48,13\right)$ mas não de interação $\left(F(3,155)=1,722, \eta_{\mathrm{p}}{ }^{2}=0,009 p=0,191\right)$. Isto indicou que apontar pontos positivos levou maior tempo, assim como a tarefa de maior dificuldade reforçando as checagens diretas. Isto também revelou que o tempo de 3 minutos foi mais que suficiente para a maioria dos respondentes e, esta maioria (aproximadamente $78 \%$ ) respondeu o quanto desejou, e apenas uma pequena parte $(22 \%)$ usou os três minutos inteiros (apenas $7 \%$ dos indivíduos no grupo de 6 fatos usaram todo o tempo e; $35 \%$ do grupo de 12 fatos).

\subsubsection{Resultados}

Testes two-way Anovas para a variável dependente média de satisfação (alpha de Cronbach 0,911) com os fatores de valência (pontos positivos vs negativos) e número de pontos (6 pontos vs 12 pontos) não revelaram interação entre os fatores $\left(F(3,155)=0,408, \eta_{\mathrm{p}}{ }^{2}=0,003\right.$ $p=0,524)$, tampouco para os grupos com diferentes números de pontos $\left(p=429, \eta_{\mathrm{p}}{ }^{2}=0,004\right)$ mas significativos para a valência manipulada $\left(p=0,009, \eta_{\mathrm{p}}{ }^{2}=0,043\right)$ (figura 1). Estes 


\section{VIESES NA SATISFAÇÃO: O EFEITO MODERADOR DA DIFICULDADE E DA} OBRIGAÇÃO SOBRE RECORDAÇÕES POSITIVAS E NEGATIVAS

resultados indicam que solicitar que os respondentes apontassem apenas pontos/fatos positivos $\left(\mathrm{M}_{\text {positivos }}=5,74 \mathrm{DP}=1,84\right)$ fez com que a satisfação média fosse maior em relação ao grupo que deveria apontar apenas fatos/pontos negativos $\left(\mathrm{M}_{\text {negaivos }}=5,01 \quad \mathrm{DP}=1,70\right)$. Entretanto, análises intra grupos utilizando post hoc com ajuste Bonferroni indicaram que a diferença era apenas significativa para o grupo que relatou 12 pontos e teve maior dificuldade $\left(\mathrm{M}_{12 \text { positivos }}=5,72 \mathrm{DP}=1,72 / \mathrm{M}_{12 \text { negativos }}=4,80 \mathrm{DP}=1,73, p=0,023 \eta_{\mathrm{p}}{ }^{2}=0,033\right)$ e não para os que relataram 6 pontos e maior facilidade $\left(\mathrm{M}_{6 \text { positivos }}=5,76 \mathrm{DP}=1,98 / \mathrm{M}_{6 \text { negativos }}=5,21 \mathrm{DP}=1,68\right.$, $p=0,160 \eta_{\mathrm{p}}{ }^{2}=0,013$ ) corroborando com a hipótese 1 . Ou seja, quanto maior a dificuldade percebida na tarefa em si, mais os respondentes ruminam e focam nas suas memórias, afetando suas avaliações.

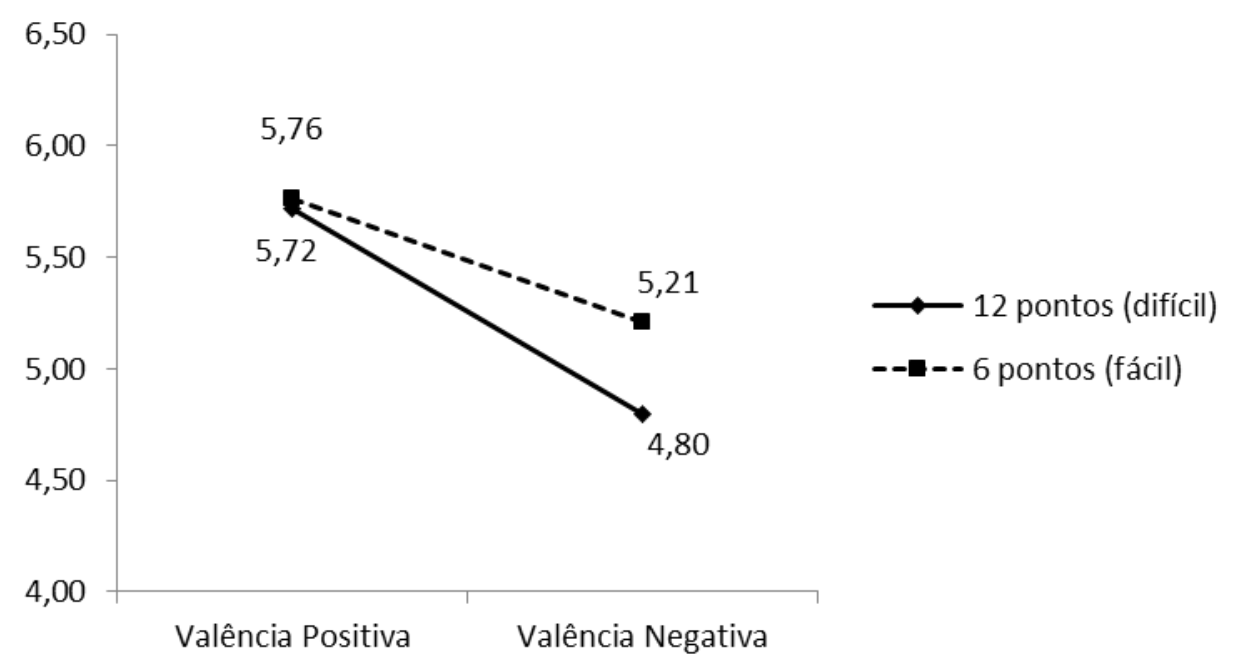

Figura 1 - Média das Avaliações de Satisfação entre os Grupos de Diferentes Valências.

Fonte: Estudo 1 dos autores.

\subsubsection{Discussão}

Os resultados do primeito estudo são congruentes com os de Winkielman e Schwarz (2001) de que, ao atribuir à dificuldade à tarefa em si (e não à memória) as diferentes valências só resultariam em diferenças significativas de avaliação quando houvesse maior dificuldade. Winkielman e Schwarz (2001) mostraram que quando as pessoas eram solicitadas a lembrar somente alguns fatos, elas deliberavam pouco e assim, não alteravam as oponiões sobre o passado. Porém, quando a dificuldade era maior, a deliberação sobre as informações também aumentava (congruente também com KAHNEMAN, 2011; NORDGREN E DIJKSTERHUIS, REAd | Porto Alegre - Edição 81 - N 2 - maio/agosto 2015 - p. 437-461 
2009; SCHWARZ, 2001), o que alterava as opiniões dos indivíduos. De fato como reportado no experimento, Kahneman (2011) aponta que quanto mais rápida, fácil ou automática uma avaliação é formada, menor tendem a ser o esforço e a deliberação de informações.

Entretanto, apesar da limitação de tempo ser utilizada para indicar que a tarefa em si seria difícil (BETTMAN e outros, 1998) as diferenças de dificuldade e tempo para resgatar memórias de valência diferentes indicam que este efeito não pode ser descartado. Isto ocorre pois lembranças negativas são mais salientes (BRAINER e outros, 1998) e assim fáceis de recordar (WINKIELMAN E SCHWARZ, 2001).

Não só o fato das avaliações de satisfação terem sido influenciadas mas, também a forma como isso foi realizado é importante. Primeiro porque, as avaliações de satisfação feitas, mesmo que pontuais, serviriam para criar expectativas de satisfação futuras (OLIVER, 2010), influenciando o boca-a-boca, recompra e a consequentemente a lealdade (BLOEMER e outros, 2007). Segundo porque, as avaliações de satisfação são carregadas de informações positivas e negativas, demonstrando que o direcionamento de que memórias serão recordadas tem efeito não só teórico, mas também prático. Ainda, se forem recordados de forma natural, mesmo consumidores satisfeitos podem ser influenciados negativamente pela saliência e facilidade de lembranças negativas. Quanto maior a facilidade, ele perceberá mais correto (KAHNEMAN, 2011) o seu próprio boca-a-boca, podendo afetar sua satisfação.

Para reforçar os resultados, e excluir a possibilidade de que o efeito de dificuldade ligado à memória (WINKIELMAN E SCHWARZ, 2001; SCHWARZ, 2004) pode ter influenciado os resultados, ao invés da deliberação em si (SCHWARZ, 2001; NORDGREN E DIJKSTERHUIS, 2009; KAHNEMAN, 2011) e, em atos de volição (PRITCHARD e outros 1999; FISCHBACK e FERGUSON, 2007) foi realizado o estudo 2. Desta forma, no segundo experimento procura-se também demonstrar que este padrão se repete quando existe o ato de volição, mas os resultados são diferentes se houver o caráter de obrigatoriedade da tarefa (hipótese 2).

\subsection{Estudo 2}

\subsubsection{Amostra e procedimentos}

REAd | Porto Alegre - Edição 81 - N 2 - maio/agosto 2015 - p. 437-461 


\section{VIESES NA SATISFAÇÃO: O EFEITO MODERADOR DA DIFICULDADE E DA} OBRIGAÇÃO SOBRE RECORDAÇÕES POSITIVAS E NEGATIVAS

Um total de 128 alunos de graduação (54,68\% homens, idade média 22 anos) participaram de experimento em salas de aula. Ele foi concebido para verificar as hipóteses 1 e 2 simultaneamente. O design do experimento foi um factorial design com 3 variáveis independentes manipuladas, resultando num 2 ("positivos" vs "negativos") x 2 (12 vs 6 fatos/pontos) x 2 ("estimulados - sem reforçar obrigação" e, "reforçando a obrigação" de completar a tarefa).

O experimento consistiu novamente numa adapatação da manipulação de Winkielman e Schwarz (2001), mas com mudanças quanto a reforçar a obrigação de completar todos os pontos/fatos para parte da amostra. Outra modificação foi a utilização da manipulação original de Winkielman e Schwarz (2001) para controlar a dificuldade em todos os grupos. Tanto para as diferenças de dificuldade percebida entre recordar pontos positivos e negativos e, para apontar que a tarefa em si era difícil (e não a recordação) restringindo o efeito da acessibildiade da memória (SCHWARZ e outros, 1991, HADDOC 2002 e AARTS E DIJKTERHUIS, 1999) como explicação. Desta forma, se houvesse alteração do padrão entre obrigar e não obrigar os estudantes a completar a tarefa, o efeito não poderia ser atribuíduo à percepção da origem da dificuldade.

Como em Winkielman e Schwarz (2001), para fatores positivos era informado aos alunos que "Algumas recordações positivas vão desaparecendo e são difíceis de recordar. Estes eventos positivos são esquecidos pois não pensamos muito sobre eles." e para a recordação de fatores negativos que "Algumas recordações negativas vão desaparecendo e são difíceis de recordar. Estes eventos são esquecidos pois as pessoas procuram esquecê-los.”

Como no experimento anterior, na primeira página da pesquisa preenchiam dados pessoais e sócio-demográficos, e em seguida os participantes eram distribuídos aleatoriamente em um de 4 grupos (12 pontos/fatos positivos, 12 pontos/fatos negativos, 6 pontos/fatos positivos ou 6 pontos/fatos negativos).

Diferente do experimento anterior, neste deveriam completar a tarefa com fatos/pontos sobre a Universidade que estudavam. Não foi imposto nenhum tempo para terminar qualquer etapa da tarefa, tampouco foi tomado o tempo (no anterior o aplicativo Qualtrics fora programado) e a manipulação da obrigatoriedade foi feita oralmente, de forma constante em metade das salas (média de 30 participantes por sala), e nas demais nada foi reforçado.

REAd | Porto Alegre - Edição 81 - № 2 - maio/agosto 2015 - p. 437-461 
A manipulação de obrigatoriedade consistia na fala "vocês não podem virar a página se não terminarem de relatar todos os fatos" alternada com "vocês devem terminar todos os fatos". Para evitar problemas na aplicação, como diferenças de tempo entre os modos de manipulação (sem obrigatoriedade levavam em média 15 minutos, com obrigatoriedade 30 minutos) e causar desistências ou outras discussões que afetassem o teste, esta manipulação de ("estimulados" vs "obrigados") não pode ser considerada aleatória. Apenas 8 respondentes não completaram as tarefas foram retirados previamente da amostra e de todas as análises.

Depois de apontar os fatos sobre a Universidade respondiam 04 questões sobre satisfação com ela como no experimento anterior. Em seguida, respondiam sobre a dificuldade percebida em apontar os pontos/fatos sobre a Universidade ("1 - muito fácil" / "10 - muito difícil") e a confiança sobre o relato dos pontos/fatos que aconteceram ("Indique a porcentagem de confiança geral de que você relatou os fatos exatamente como acontece/ocorreram com você").

\subsubsection{Checagem das manipulações}

Os resultados das checagens foram considerados satisfatórios. Testes $t$ indicaram maior número de fatos/pontos para o grupo com 12 pontos $(\mathrm{n}=66)$ do que para o grupo de 6 pontos $(\mathrm{n}=62) \quad\left(t=6,860 \quad p<0,001 ; \quad \mathrm{M}_{12 \text { pontos }}=7,90 \quad \mathrm{DP}=2,84 ; \quad \mathrm{M}_{6 \text { pontos }}=5,22 \quad \mathrm{DP}=1,20\right) \quad \mathrm{e}$ maior dificuldade em apontar fatos $\left(t=2,446 p=0,016 ; \mathrm{M}_{12 \text { pontos }}=5,54 \mathrm{DP}=1,91 ; \mathrm{M}_{6 \text { pontos }}=4,74\right.$ $\mathrm{DP}=1,71)$, mas sem diferenças para a média de satisfação (alpha de Cronbach 0,791) $(t=0,246$ $\left.p=0,806 ; \mathrm{M}_{12 \text { pontos }}=6,51 \mathrm{DP}=1,19 ; \mathrm{M}_{6 \text { pontos }}=6,57 \mathrm{DP}=1,26\right)$. Foi verificada uma diferença marginal para confiança dos fatos que relataram entre estes grupos $(t=1,183 p=0,071$; $\mathrm{M}_{12 \text { pontos }}=80,83 \mathrm{DP}=24,74 ; \mathrm{M}_{6 \text { pontos }}=87,43 \mathrm{DP}=14,62$ ) no sentido esperado, já que a maior dificudaldade diminuiria a confiança.

Acessoriamente, testes $t$ indicaram que respondentes que tiveram que apontar apenas pontos positivos $(n=61)$ não tiveram diferenças com os demais (negativos, $n=67$ ) quanto ao número de pontos/fatos $\left(t=1,086 p=0,280 ; M_{\text {pontosnegativos }}=6,37 \mathrm{DP}=2,45 ; \mathrm{M}_{\text {pontospositivos }}=6,86\right.$ $\mathrm{DP}=2,71)$ e tampouco perceberam maior dificuldade em relatar fatos/pontos do que aqueles que apontaram apenas pontos negativos $\left(t=1,333 p=0,185 ; \quad \mathrm{M}_{\text {pontosnegativos }}=4,94 \mathrm{DP}=2,02\right.$; $\mathrm{M}_{\text {pontospositivos }}=5,37 \mathrm{DP}=1,63$ indicando que o controle de dificuldade entre fatos positivos e negativos obteve êxito.

REAd | Porto Alegre - Edição 81 - № 2 - maio/agosto 2015 - p. 437-461 


\section{VIESES NA SATISFAÇÃO: O EFEITO MODERADOR DA DIFICULDADE E DA} OBRIGAÇÃO SOBRE RECORDAÇÕES POSITIVAS E NEGATIVAS

Como no experimento anterior houve diferença para a média de satisfação $(t=4,091$ $\left.p<0,001 ; \mathrm{M}_{\text {pontosnegativos }}=6,14 \mathrm{DP}=1,24 ; \mathrm{M}_{\text {pontospositivos }}=6,98 \mathrm{DP}=1,04\right)$ e para a confiança dos relatos $\left(t=3,532 p<0,001 ; \mathrm{M}_{\text {pontosnegativos }}=89,92 \mathrm{DP}=14,93 ; \mathrm{M}_{\text {pontospositivos }}=77,55 \mathrm{DP}=24,01\right)$ ao manipular a valência das memórias requisitada. Desta forma, verificamos pelos dois experimentos que mesmo aplicando os procedimentos de controle sobre dificuldade da tarefa (BETTMAN e outros, 1998; WINKIELMAN E SCHWARZ, 2001), memórias negativas estiveram atreladas à maior confiança.

Testes $t$ indicaram que respondentes que tiveram "reforços de obrigatoriedade constantes" ( $\mathrm{n}=70)$ tiveram diferenças com os demais ("sem reforço de obrigatoriedade", $\mathrm{n}=58$ ) quanto ao número de pontos/fatos ( $t=2,260 p=0,026 ; \mathrm{M}_{\text {origatoriedade }}=7,07 \mathrm{DP}=2,60$; $\left.\mathrm{M}_{\text {semobrigatoriedade }}=6,05 \mathrm{DP}=2,46\right)$ e também percepção marginal de maior dificuldade em relatar fatos/pontos $\left(t=1,872 p=0,064 ; \mathrm{M}_{\text {obrigatoriedade }}=5,48 \mathrm{DP}=1,87 ; \mathrm{M}_{\text {semobrigatoriedade }}=4,87 \mathrm{DP}=1,80\right)$, mas sem diferenças para as médias de satisfação $\left(t=1,327 p=0,187 ; \mathrm{M}_{\text {obrigatoriedade }}=6,41\right.$ $\left.\mathrm{DP}=1,37 ; \mathrm{M}_{\text {semobrigatoriedade }}=6,70 \mathrm{DP}=1,01\right)$ ou para a confiança dos relatos $(t=0,865 p=0,388$; $\left.\mathrm{M}_{\text {obrigatoriedade }}=85,47 \mathrm{DP}=19,54 ; \mathrm{M}_{\text {semobrigatoriedade }}=82,29 \mathrm{DP}=21,98\right)$.

Análises dentro dentro destes grupos não indicaram interações entre as valências e a quantidade de fatos (sem reforço de obrigatoriedade: $\left(F(3,54)=0,368, \eta_{\mathrm{p}}{ }^{2}=0,007 p=0,547\right)$ / reforço de obrigatoriedade $\left.\left(F(3,66)=0,091, \eta_{\mathrm{p}}{ }^{2}=0,001 p=0,764\right)\right)$ sobre a confiança. Análsies post hoc com ajuste Bonferroni indicaram que a única diferença significativa para a confiança entre grupos nestas análises foi para as diferentes valências dentro do grupo que foi obrigado a completar a tarefa $\left(p=0,001, \eta_{\mathrm{p}}^{2}=0,007, \quad \mathrm{M}_{\text {negativos }}=93,33 \quad \mathrm{DP}=11,65, \quad \mathrm{M}_{\text {positivos }}=77,15\right.$ $\mathrm{DP}=22,71$ ). Entretanto, estes resultados exclui a explicação causal de que a falta de confiança tenha afetado os julgamentos (HADDOCK, 2002, AARTS E DIJKTERHUIS, 1999), KAHNEMAN, 2011) entre os grupos de diferentes obrigações ("estimulados" vs "obrigados") e, tampouco de diferentes quantidades de recordações (12 vs 6 fatos/pontos).

\subsubsection{Resultados}

Testes two-way Anova sobre a variável dependente média de satisfação com os fatores valência (pontos positivos vs negativos) e número de pontos (6 pontos vs 12 pontos) não revelaram interação significativa $\left(F(3,124)=2,448, \eta_{\mathrm{p}}{ }^{2}=0,019 p=0,120\right)$, tampouco efeito para os grupos com diferentes números de pontos $\left(p=0,783, \eta_{\mathrm{p}}{ }^{2}=0,001\right)$ mas significativos REAd | Porto Alegre - Edição 81 - N 2 - maio/agosto 2015 - p. 437-461 
Juan José Camou Viacava \& Danielle Mantovani

para a valência manipulada $\left(p<0,001, \eta_{\mathrm{p}}{ }^{2}=0,019\right)$. Como no primeiro experimento, estes resultados indicam que solicitar que os respondentes apontassem apenas pontos/fatos positivos elevou a satisfação média em relação ao grupo que deveria apontar apenas fatos/pontos negativos $\left(\mathrm{M}_{\text {pontosnegativos }}=6,14 \mathrm{DP}=1,24 ; \mathrm{M}_{\text {pontospositivos }}=6,98 \mathrm{DP}=1,04\right)$.

Teste three way Anova das três variáveis independentes indicou uma interação entre elas $\left(F(7,120)=4,965, \eta_{\mathrm{p}}{ }^{2}=0,040 p=0,028\right)$ e suporte inicial à hipótese 2. Pela recomendação de Keppel e Wickin (2004), análises two way Anova foram realizadas separadamente dentro dos dois diferentes grupos (“estimulados" vs “obrigados”) para verificar os resultados.

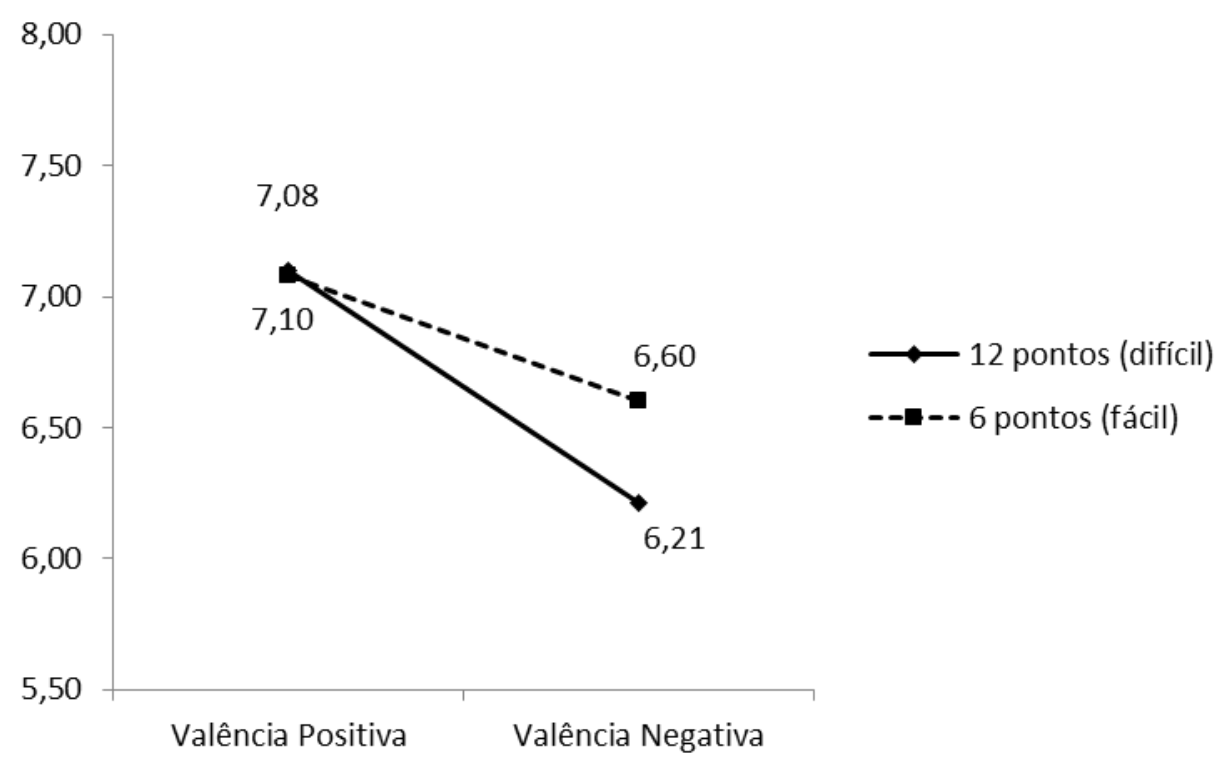

Figura 2 - Média das Avaliações de Satisfação entre os grupos, para indivíduos "estimulados" a completar a tarefa.

Fonte: Estudo 2 dos autores.

No grupo que não houve reforço de obrigatoriedade não foi verificada interação $\left(F(3,54)=0,638, \eta_{\mathrm{p}}^{2}=0,012 p=0,428\right)$ (figura 2). Análises intra grupos utilizando post hoc com ajuste Bonferroni indicaram não haver diferenças entre 6 pontos positivos ou negativos $\left(\mathrm{M}_{6 \text { pontosnegativos }}=6,60 \mathrm{DP}=0,84 ; \mathrm{M}_{6 \text { pontospositivos }}=7,08 \mathrm{DP}=0,74 p=0,227\right)$ mas com diferenças significativas entre os grupos com 12 pontos $\left(\mathrm{M}_{12 \text { pontosnegativos }}=6,21 \quad \mathrm{DP}=1,19\right.$; $\mathrm{M}_{12 \text { pontospositivos }}=7,10 \mathrm{DP}=0,83 p=0,010$ ), congruente com o experimento 1 e corroborando a hipótese 1. Dentro dos grupos de diferentes valências não foram encontrados resultados com diferenças significativas entre as dificuldades (negativa $p=0,271 /$ positivo $p=0,964$ ).

REAd | Porto Alegre - Edição 81 - N 2 - maio/agosto 2015 - p. 437-461 


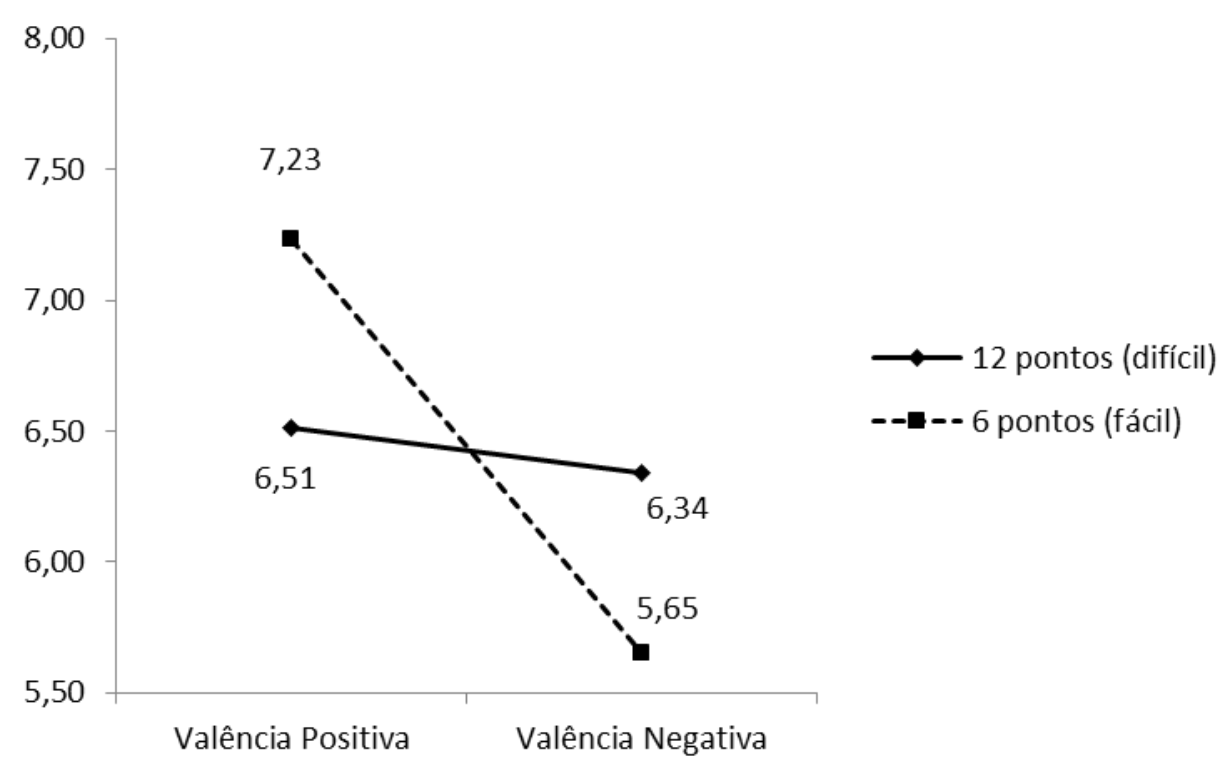

Figura 3 - Média das Avaliações de Satisfação entre os grupos, para indivíduos "obrigados" a completar a tarefa Fonte: Estudo 2 dos autores.

No grupo que houve reforço de obrigatoriedade foi verificada uma interação $\left(F(3,66)=5,406, \eta_{\mathrm{p}}{ }^{2}=0,076 p=0,023\right)$ (figura 3). Análises intra grupos utilizando post hoc com ajuste Bonferroni indicaram haver diferenças significativas entre 6 pontos positivos / negativos $\left(\mathrm{M}_{6 \text { pontosnegativos }}=5,65 \mathrm{DP}=1,35 ; \mathrm{M}_{6 \text { pontospositivos }}=7,23 \mathrm{DP}=1,11 p<0,001\right)$ mas sem diferenças significativas entre os grupos com 12 pontos $\left(M_{12 \text { pontosnegativos }}=6,34 \mathrm{DP}=1,31\right.$; $\left.\mathrm{M}_{12 \text { pontospositivos }}=6,51 \mathrm{DP}=1,25 \mathrm{p}=0,702\right)$. Dentro dos grupo de diferentes valências foram encontrados resultados marginais (negativa $p=0,107$ / positivo $p=0,103$ ).

\subsubsection{Discussão}

Mais uma vez aqui o padrão de que, sob atos de volição a dificuldade interage a com as diferentes valências emerge dos dados. Ainda, com os procedimentos para atribuir a dificuldade à tarefa em todos os grupos ficou corroborada a hipótese 2. Demonstrou-se não só que a percepção de origem de dificuldade da tarefa afeta os resultados, mas também quanto ao ato de volição.

Com exceção destes resultados marginais que podem atribuídos à pequena amostra (FIELD, 2009), estes resultados no grupo de obrigatoriedade são similares com os resultados 
de Schwarz e outros (1991), Haddock (2002), Aarts e Dijkterhuis (1999). Entretanto, as checagens aqui demonstram que o efeito não pode ser atribuído à variação de confiança, alterando os julgamentos (HADDOCK, 2002; AARTS E DIJKTERHUIS, 1999; KAHNEMAN, 2011), e sim apenas ao restringir a vontade dos participantes, exigindo que eles terminem a tarefa (PRITCHARD e outros 1999; BAGOZZI e LEE, 1999; KORIAT e outros, 2008).

\section{CONSIDERAÇÕES FINAIS}

Os experimentos corroboraram com outros estudos ao demonstrar haver uma ligação entre as avaliações de satisfação, fatos recordados e, a facilidade em relatá-los. Memórias e avaliações negativas geram maior recordação e maior facilidade, (KANOUSE E HANSON, 1972; KANOUSE, 1984; BRAINERD e outros, 2010) e a facilidade está relacionada à maior confiança (KAHNEMAN, 2011). Desta forma, foram encontradas evidências pelo comportamento análogo de boca-a-boca que clientes insatisfeitos propagam mais informações e tem mais confiança no processo.

Além de demonstrar estes efeitos, os experimentos corroboraram as hipóteses 1 e 2, que além de sua relevância teórica também demonstram formas práticas de enviesar as avaliações de satisfação em dois ramos de serviços. De forma geral, estimular relatos de fatos positivos (negativos) enviesa positivamente (negativamente) as avaliações. Entretanto, os resultados dependem do grau de dificuldade percebida na tarefa em si e, também da obrigatoriedade em completar a tarefa. Quando não houve obrigatoriedade e os respondentes apresentavam fatos/pontos quanto achassem necessários, houve apenas diferenças significativas entre apontar fatos positivos e negativos quando a dificuldade foi de maior intensidade (semelhante à WINKIELMAN E SCHWARZ, 2001). Quando foi manipulada a obrigatoriedade, mesmo que não fosse alcançado o número exato de fatos, o efeito foi diferente (semelhante aos resultados de HADDOCK, 2002 e, AARTS E DIJKTERHUIS, 1999). Entretanto, verificou-se que este efeito não estaria tanto ligado à diferenças na confiança (KORIAT e outros, 2008), mas sim pelo impacto da volição (BAGOZZI, 1993; PRITCHARD e outros 1999; FISCHBACK e FERGUSON, 2007; HIGGINS e SCHOLER, 2009).

REAd | Porto Alegre - Edição 81 - № 2 - maio/agosto 2015 - p. 437-461 


\section{VIESES NA SATISFAÇÃO: O EFEITO MODERADOR DA DIFICULDADE E DA} OBRIGAÇÃO SOBRE RECORDAÇÕES POSITIVAS E NEGATIVAS

Esta pesquisa apresentou uma relação indireta com possíveis falsas recordações e lembranças (lures) nos relatos. Isto ocorre pois foi aberta a possibilidade dos respondentes relatassem fatos sobre suas empresas prestadoras de serviços, mas não foram feitos testes para verificar se as memórias eram de fato verdadeiras. Neste sentido, Pezdek, Finger, e Hodge (1997) apontaram que eventos plausíveis de alta probabilidade de ocorrência (como se perder momentaneamente no shopping) levariam a mais falsas memórias do que não plausíveis (ter tido uma lavagem intestinal - ambos exemplos do autor), ainda que Talarico e Rubin (2003) apontem que informações de valência negativa aumentam a confiança e a nitidez subjetiva sem afetar a precisão em testes de memória. Entretanto, Brainerd e outros (2010) apontam que informações de valência negativa (como fatos negativos tão comuns do setor de telefonia) levam a mais casos de lures, pela tendência de incorporação de eventos similares (LYLE e JOHNSON, 1995).

Os estudos 1 e 2 demonstraram formas de enviesamento nas avaliações de satisfação, mas outros mecanismos também podem afetar o processamento cognitivo. Trudel e Cotte (2012) demonstraram que participantes sob o foco regulatório (um modo de autocontrole, BARON, 2008) de promoção (focados em aproximar-se de ganhos e aspectos positivos como referências) relataram níveis mais elevados de satisfação que indivíduos em prevenção (focados em evitar perdas, aspectos negativos como referências) ao avaliar produtos com desconfirmanção positiva, enquanto indivíduos em prevenção relataram maior satisfação em situações de desconfirmação negativa. Similarmente Zhang e Cheng (2013) demonstraram diferentes efeitos dos focos regulatórios sobre recuperação de trangressões (overbooking) afetando a satisfação no setor de aviação. Neste, indivíduos em prevenção (vs promoção) ficavam mais insatisfeitos com a falta de atributos de recuperação obrigatórios/funcionais (classificados como must-be: reembolso de passagens e desculpas dos empregados), mas não aumentaram sua satisfação quando estavam disponíveis (teoricamente, alcançavam seu "teto", como no modelo de KANO, 1984). Assim, não só o processamento cognitivo pode ser afetado pela facilidade/dificuldade de avaliação e resgate de memórias, saliência, quantidade, valência e/ou obrigatoriedade, mas outros mecanismos como o próprio autocontrole e autorregulação podem afetar as avaliações. Passar a entender de forma mais completa e como diferentes mecanismos atuam em conjunto, como motivações, cognição, emoções e significados, ainda parece ser uma forma de redescobrir a satisfação (FOURNIER E MICK, 1999).

REAd | Porto Alegre - Edição 81 - № 2 - maio/agosto 2015 - p. 437-461 


\section{REFERÊNCIAS}

AKER, J. Dimensions of Brand Personality. Journal of Marketing Research, v. 34, p. 347356, 1997.

AARTS, H.; DIJKSTERHUIS, A. How often did I do it? Experienced ease of retrieval and frequency estimates of past behavior. Acta Psychologica, v. 103, p. 77-89, 1999.

ARNDT, J.; REDER, L. M. The effect of distinctive visual information on false recognition. Journal of Memory and Language, v. 48, p.1-15, 2003.

RICHARD P. BAGOZZI e KYU-HYUN LEE. Consumer Resistance To, and Acceptance Of, Innovations, em NA - Advances in Consumer Research Volume 26, eds. Eric J. Arnould and Linda M. Scott, Provo, UT : Association for Consumer Research, Pages: 218-225, 1999.

BETTENCOURT, L.A. Customer Voluntary Performance: Customer as partners in service delivery. Journal of Retailing, v.73, n.3, p.383-406, 1997.

BETTMAN, J., LUCE, M. F.; PAYNE, J. W. Constructive Consumer Choice Processes, Journal of Consumer Research, v. 25 (December), p.187-217, 1998.

BLOEMER, J.; ODEKERKEN-SCHRÖDER, G. e MARTES, H. The psychology behind commitment and loyalty: an empirical study in a bank setting. Journal of Relationship Marketing, v.6(1), 21-43, 2007

BRAINERD, C. J.; HOLLIDAY, R. E.; REYANA, V. F.;YANG, Y.;TOGLIA, M. P. Developmental reversals in false memory: Effects of emotional valence and arousal. Journal of Experimental Child Psychology, v. 107, p. 137-154, 2010.

DIJKSTERHUIS, A.; BOS, M.W.; NORDGREN, L.F; VAN BAAREN, R.B. On Making the right Choice: The Deliberation-Without-Attention Effect. Science, v.311, n.17, p.1005-1007, 2006.

FORNELL, C.; JOHNSON, M.D.; AANDERSON, E.W.; CHA, J.; BRYANT, B.E. The american customer satisfaction: Nature, purpose and findings. Journal of Marketing, v.58, n.4, p.7-18, 1994.

FISCHBACK, A. e FERGUSON, M. J. (2007) The goal construct in social psychology. Em KRUGLANKI, A.W. e HIGGINS, T.E (Eds). Social Psychology: Handbook of Basic Principles.

REAd | Porto Alegre - Edição 81 - № 2 - maio/agosto 2015 - p. 437-461 

OBRIGAÇÃO SOBRE RECORDAÇÕES POSITIVAS E NEGATIVAS

FOURNIER, S.; MICK, D. G. Rediscovering Satisfaction. Journal of Marketing, v.63, p.523, October, 1999.

HADDCOCK, G. It's easy to like or dislike Tony Blair: Accessibility experiences and the favourability of attitude judgments. British Journal of Social Psychology, v. 93, p. 257-267, 2002.

HART, C.W.; JOHNSON, M.D. Growing the trust relationship. Marketing Management. p.919, 1999.

HOMMEL, B., MÜSSELER, J.; ASCHERSLEBEN, G.; PRINZ, W. The theory of event coding (TEC): A framework for perception and action planning. Behavioral and Brain Sciences, v.24, p.849-878, 2001.

HIGGINS, E.T. e SCHOLER, A. (2009) Engaging the consumer: The science and art of the value creation process. Journal of Consumer Psychology, Research Dialogue, v. 19, p.100114.

GARBARINO, E.; JOHNSON, M. S. The different roles of satisfaction, trust and commitment in consumer relationships. Journal of Marketing, v.3, p. 70-97, 1999.

GOODWIN, C. J. Research in Psychology: Methods and Design. [s. 1.]: Wiley, 2008.

GOMES, C. F.A.; BRAINED, C. J.; STEIN, L. M. Effects of Emotional Valence and Arousal on Recollective and Nonrecollective Recall. Journal of Experimental Psychology: Learning, Memory, and Cognition, v. 39, n.3, p.663-677, 2013.

JOHNSON, M. K. False memories, Psychology of. In: SMELSER, N. J.; BALTES, P.B. (Eds.). International Encyclopedia of the Social \& Behavioral Sciences (V. 8). Amsterdam, New York: Elsevier Science, 2001. p. 5254-5259.

KAHNEMAN, D. Rápido e Devagar: Duas Formas de Pensar. Rio de Janeiro: Objetiva, 2011.

KANO, N.; SERAKU, N.; TAKAHASHI, F.; TSUJI, S. Attractive quality and must be quality. Hinshitsu, The Journal of the Japanese Society for Quality Control, p. 39-48, 1984. 
Juan José Camou Viacava \& Danielle Mantovani

KELLER, K. L. Gestão Estratégica de Marcas. São Paulo: Pearson Prentice Hall, 2006.

KEININGHAM, T. L.; COOIL, B.; ANDREASSEN, T. W.; AKSOY, L. A Longitudinal Examination of Net Promoter and Firm Revenue Growth. Journal of Marketing, v. 71, p. 39$51,2007$.

KANOUSE, D. E.; HANSON, L. R. Negativity in Evaluations. In: JONES, Edward E, KANOUSE, D. E.; KELLEY, H.H.; NISBETT, R. E.; VALINS, S.; WEINER, B. Attribution: Perceiving the Causes of Behaviour. Morristown, Nova York: General Learning Press, 1972. p. $47-62$.

. Explaining Negativity Biases in Evaluation and Choice Behavior: Theory and Research. Advances in Consumer Research, v.11, p.703-708, 1984. KINNEAR, T. C. (Ed.) Association for Consumer Research.

KEPPEL, G.; WICKINS, T. D. Design and Analysis: A Researcher's Handbook (4. ed.). Upper Saddle River, NJ: Prentice Hall, 2004.

LOFTUS, E.; PICKRELL, J. The formation of false Memories. Psychiatric Annals, v. 25, 12, p. $720-725,1995$.

LYLE, K. B.; JOHNSON, M.K. Importing perceived memories into false memories. Memory, v.14, n. 2, p.197-213, 2006. Psychology Press - Taylor Francis Group.

NORDGREN, L. F.; DIJSTERHUIS, A. The Devil Is in the Deliberation: Thinking Too Much Reduces Preference Consistency. Journal of Consumer Research, v. 36, June, p. 39-46, 2009.

OLIVER, Richard L. A Cognitive Model of the Antecedents and Consequences of Satisfaction Decisions. Journal of Marketing Research, v.17, p. 460-469, 1980.

. Cognitive, Affective and Attribute Bases of the Satisfaction Response.

Journal of Consumer Research, v.20, p. 418-430, 1993.

. Whence Consumer Loyalty? Journal of Marketing, v. 63, p.33-44, 1999.

Fundamental Issues and Directions for Marketing.

REAd | Porto Alegre - Edição 81 - N 2 - maio/agosto 2015 - p. 437-461 
. Satisfaction: a behavioral perspective on the consumer. (1. ed.).(2. ed.).

New York: M. E. Sharpe, 2010 [1997].

PEZDEK, K.; FINGER, K.; HODGE, D. Planting false childhood memories: The role of event plausibility. Psychological Science, v. 8, p. 437-441, 1997.

PRADO, P. H. M.; SANTOS, R. C. Satisfação, qualidade no relacionamento e lealdade entre Clientes e bancos de varejo. X Encontro de Marketing da ANPAD, 2004. Anais. Rio de Janeiro: ANPAD, 2004.

PRITCHARD, M.P; HAVITZ, M.E. e HOWARD, D.R. Analyzing the commitment-loyalty link in Service Contexts. Journal of Academy of Marketing Service, v.27(3), p.333-48, 1999. ROTELLO , C.M.;MACMILLAN, N.A.; HICKS, J. e HAUTS, M. Interpreting the effects of response bias on remember-know judgments using detection and threshold models. Memory and Cognition, v.34 (8), p. 1598-1614, 2006.

SIDDIGI, K.O. Interrelations between service quality attributes, customer satisfaction and customer loyalty in the retail banking sector in Bangladesh. International Trade \& Academic Research Conference (ITARC) - London 2010, v.1, p. 1-26, 2010.

SCHWARZ, N.; BLESS, H; STRACK, F.; KLUMPP, G.; RITTENNAUER-SCHATKA, H.; SIMMONS, A. Ease o retrieval as information: Another look at the availability heuristic. Journal of personality and Social Psychology, v. 61, p. 195-202, 1991.

SZPUNAR, K.; ADDIS, D. R.; SCHACTER, D. L. Memory for Emotional Simulations: Remembering a Rosy Future. Psychological Science v.23, p.24-29, 2012.

TALARICO, J. M.; RUBIN, D. C. Confidence, not consistency, characterizes flashbulb memories. Psychological Science, v. 14, p. 455-461, 2003.

TRUDEL, R., MURRAY, K.; COTTE, J. Beyond expectations: The effect of regulatory focus on consumer satisfaction. International Journal of Research in Marketing, v. 29, p. 93-97, 2012 .

TVERSKY, A.; KAHNEMAN, D. Availability: A Heuristic for Judging Frequency and Probability. Cognitive Psychology, v. 4, p. 207-232, 1973. 
WINKIELMAN, P. e SCHWARZ, N. How pleasant was your childhood? Beliefs about memory shape inferences from experienced difficulty of recall. Psychological Science, v. 12, p. 176-179, 2001.

ZWICK, R., PIETERS, R. e BAUGMGARTNER, H. On the Practical Significance of Highsight Bias: The Case of Expectancy-Disconfirmation Model of Consumer Satisfaction. Organizational Behaviour and Human Decision Processes, v. 64, n. 1, October, p. 103-117, 1995.

ZHANG, X.; CHENG, R. Asymmetric effects, regulatory focus, and attribute satisfactionMixed experimental evidence in airline overbooking recovery. International Journal of Production Economics. v.142, Issue1, March, p. 27-36, 2013. Special Issue on Behavioral Operations Management/Production Economics.

KORIAT, A.; NUSSINSON, R.; BLESS, H.; SHAKED, N. Information-based and experienced-based metacognitive judgments. Evidence form subjective confidence. In: DUNLOSKY, J.; BJORK, R. (Ed.). Handbook of Metamemory and Memory. New York: Taylor \& Francis Group, 2008. p. 117-135. 